\title{
Computer Visualization of Turbulent Parameter Fields in a Round Jet Based on Laser Doppler and Fiber Optics Measurements
}

\author{
V.I. Smirnov ${ }^{1}$ \\ National Research University "MPEI" \\ ${ }^{1}$ ORCID: 0000-0002-3047-988X, smirnovvi-mpei@yandex.ru
}

\begin{abstract}
$\underline{\text { Abstract }}$
Based on local laser Doppler and fiber-optic anemometric (LDA and FOA) measurements, a computer visualization of the fields of turbulence parameters in an axisymmetric submerged fluid jet flow at Reynolds number 15,000 was performed by the parametric identification method. The scheme of the FOA measurement, the instrument function of the FOA and the problem of its elimination are considered. Formulas for calculating the first four moments of the two-dimensional probability density of turbulent pulsations of the local velocity are given, taking into account instrument distortions. Simple parametric models are proposed to describe the fields of turbulence parameters in an axisymmetric jet flow. On this basis, the patterns of the average velocity field, the turbulence intensity field, and the shear stress field are reconstructed. Estimates of the coefficients of asymmetry and kurtosis of the probability density function of the local velocity are obtained based on calculations of the higher statistical moments of turbulent pulsations of the local velocity. The results of measurements of the coefficients of asymmetry and kurtosis indicate deviations of the velocity pulsation statistics from the normal law, even for axial regions at long distances from the nozzle. This may be due to the presence of coherent vortex structures in the flow and intermittency. In the peripheral areas, return flows can also affect, caused by the peculiarities of the geometry of the cuvette and its final dimensions. The presented results of quantitative visualization allow us to calculate the energy dissipation and other integral characteristics of the flow.
\end{abstract}

Keywords: laser measurements, turbulent flow, round jet, computer visualization, fields of turbulence parameters.

\section{Introduction}

The study of turbulent jets is of considerable practical and theoretical interest $[1,2]$. When developing new methods for measuring turbulence characteristics in liquid and gas flows, these objects were widely used for testing equipment, representing a variety of flow modes. A large volume of these data obtained by laser Doppler anemometry (LDA) is presented in the traditional format in [3]. Some interesting results were published in $[4,5]$.

The model of a turbulent axisymmetric submerged jet with a profiled nozzle was used also for testing equipment and for developing a method of fiber-optic anemometry (FOA) [6-9].

The LDA method is widely represented in the scientific literature, which cannot be said about the FOA method. Therefore, it would be useful to briefly outline the principles and features of the work of the FOA. 


\section{The principle of operation of the projection type FOA}

The scheme of the projection-type FOA is shown in Fig. 1. The measurement principle is based on photographing with a long exposure time the image of the luminous end of the fiber-optic sensor introduced into the investigated region of the turbulent flow.

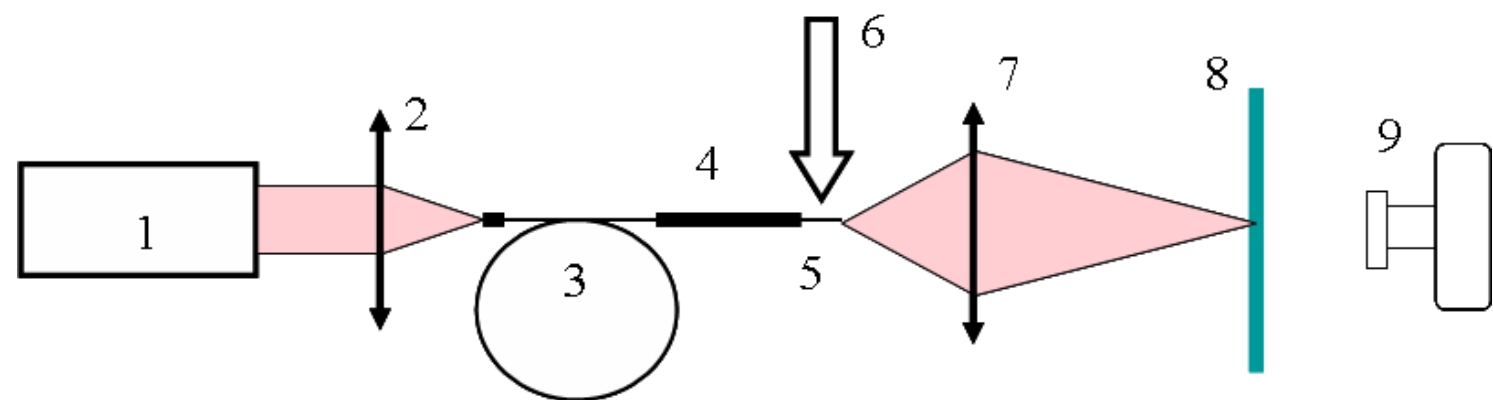

Fig. 1. The scheme of a projection-type OVLA: 1 - a semiconductor laser; 2 - a focusing optical system; 3 - an optical fiber; 4 - a frame of a sensitive element; 5 - a sensor (a sensitive element of an optical fiber); 6 - the flow under study; 7 - a projection lens; 8 - a screen; 9 - a digital camera with an interference filter.

Photographing is carried out with a large averaging time compared to the time scale of turbulence. The resulting picture is a blurred image of the radiating end in the form of a spot with a brightness distribution corresponding to the relative residence time of the end in this area. After excluding distortions associated with the final dimensions of the end face, this allows us to estimate the two-dimensional probability density of its displacement.

\section{Methods and algorithms of data processing}

Using Mathcad programming tools, the photographs were represented as a matrix of values of the relative probability of displacements of the sensor end. The following statistical parameters were calculated using standard algorithms.

The moments of the 1st and 2nd orders and the correlation coefficient $R_{\mathrm{xy}}$ :

$$
\begin{gathered}
\alpha_{x}=\bar{x}, \alpha_{y}=\bar{y}, \\
\mu_{x x}=\left\langle(x-\bar{x})^{2}\right\rangle, \quad \mu_{y y}=\left\langle(y-\bar{y})^{2}\right\rangle, \\
\mu_{x y}=\langle(x-\bar{x})(y-\bar{y})\rangle, \\
R_{x y}=\frac{\mu_{x y}}{\sqrt{\mu_{x x} \mu_{y y}}} .
\end{gathered}
$$

Moments of the $3^{\text {th }}$ order and related dimensionless shape coefficients (asymmetry coefficients):

$$
\begin{gathered}
\mu_{x x x}=\left\langle(x-\bar{x})^{3}\right\rangle, \quad \mu_{y y y}=\left\langle(y-\bar{y})^{3}\right\rangle, \\
\mu_{x x y}=\left\langle(x-\bar{x})^{2}(y-\bar{y})\right\rangle, \quad \mu_{x y y}=\left\langle(x-\bar{x})(y-\bar{y})^{2}\right\rangle, \\
\gamma_{x x x}=\frac{\mu_{x x x}}{\sqrt{\mu_{x x}^{3}}}, \gamma_{y y y}=\frac{\mu_{y y y}}{\sqrt{\mu_{y y}^{3}}}, \quad \gamma_{x x y}=\frac{\mu_{x x y}}{\sqrt{\mu_{x x}^{2} \mu_{y y}}}, \gamma_{x y y}=\frac{\mu_{x y y}}{\sqrt{\mu_{x x} \mu_{y y}^{2}}} .
\end{gathered}
$$


Moments of the 4th order and related dimensionless coefficients of the form (kurtosis coefficients):

$$
\begin{gathered}
\mu_{x x x x}=\left\langle(x-\bar{x})^{4}\right\rangle, \quad \mu_{y y y y}=\left\langle(y-\bar{y})^{4}\right\rangle, \mu_{x x x y}=\left\langle(x-\bar{x})^{3}(y-\bar{y})\right\rangle, \\
\mu_{x y y y}=\left\langle(x-\bar{x})(y-\bar{y})^{3}\right\rangle, \mu_{x x y y}=\left\langle(x-\bar{x})^{2}(y-\bar{y})^{2}\right\rangle, \\
\gamma_{x x x x}=\frac{\mu_{x x x x}}{\mu_{x x}^{2}}-3 \quad \gamma_{y y y y}=\frac{\mu_{y y y y}}{\mu_{y y}^{2}}-3 \\
\gamma_{x x x y}=\frac{\mu_{x x x y}}{\sqrt{\mu_{x x}^{3} \mu_{y y}}-3 R_{x y} \quad \gamma_{x y y y}=\frac{\mu_{x y y y}}{\sqrt{\mu_{x x} \mu_{y y}^{3}}}-3 R_{x y}}, \quad, \quad \\
\gamma_{x x y y}=\frac{\mu_{x x y y}}{\mu_{x x} \mu_{y y}}-\left(1+2 R_{x y}^{2}\right)
\end{gathered}
$$

All coefficients of the form are defined in such a way that they are reset to zero for a normal distribution.

The estimation biases due to the finite dimensions of the sensor for a symmetric instrument function were taken into account according to the formulas:

$$
\begin{aligned}
& R_{x y}^{T}=R_{x y}^{M} \sqrt{\frac{\mu_{x x}^{M} \mu_{y y}^{M}}{\left(\mu_{x x}^{M}-\mu_{x x}^{A}\right)\left(\mu_{y y}^{M}-\mu_{y y}^{A}\right)}} \\
& \gamma_{x x x}^{T}=\gamma_{x x x}^{M}\left(\frac{\mu_{x x}^{M}}{\mu_{x x}^{M}-\mu_{x x}^{A}}\right)^{3 / 2}, \quad \gamma_{y y y}^{T}=\gamma_{y y y}^{M}\left(\frac{\mu_{y y}^{M}}{\mu_{y y}^{M}-\mu_{y y}^{A}}\right)^{3 / 2}, \\
& \gamma_{x x y}^{T}=\gamma_{x x y}^{M} \sqrt{\frac{\left(\mu_{x x}^{M}\right)^{2}\left(\mu_{y y}^{M}\right)}{\left(\mu_{x x}^{M}-\mu_{x x}^{A}\right)^{2}\left(\mu_{y y}^{M}-\mu_{y y}^{A}\right)}} \\
& \gamma_{x y y}^{T}=\gamma_{x y y}^{M} \sqrt{\frac{\left(\mu_{x x}^{M}\right)\left(\mu_{y y}^{M}\right)^{2}}{\left(\mu_{x x}^{M}-\mu_{x x}^{A}\right)\left(\mu_{y y}^{M}-\mu_{y y}^{A}\right)^{2}}} \\
& \gamma_{x x x x}^{T}=\gamma_{x x x x}^{M}\left(\frac{\mu_{x x}^{M}}{\mu_{x x}^{M}-\mu_{x x}^{A}}\right)^{2}-\gamma_{x x x x}^{A}\left(\frac{\mu_{x x x x}^{A}}{\mu_{x x x x}^{M}-\mu_{x x x x}^{A}}\right)^{2} \text {, } \\
& \gamma_{y y y y}^{T}=\gamma_{y y y y}^{M}\left(\frac{\mu_{y y}^{M}}{\mu_{y y}^{M}-\mu_{y y}^{A}}\right)^{2}-\gamma_{y^{4}}^{A}\left(\frac{\mu_{y y}^{A}}{\mu_{y y}^{M}-\mu_{y y}^{A}}\right)^{2} \\
& \left.\gamma_{x x y y}^{T}=\gamma_{x x y y}^{M} \frac{\mu_{x x}^{M} \mu_{y y}^{M}}{\left(\mu_{x x}^{M}-\mu_{x x}^{A}\right)\left(\mu_{y y}^{M}-\mu_{y y}^{A}\right.}\right)^{-\gamma_{x x y y}^{A}} \frac{\mu_{x x}^{A} \mu_{y y}^{A}}{\left(\mu_{x x}^{M}-\mu_{x x}^{A}\right)\left(\mu_{y y}^{M}-\mu_{y y}^{A}\right)},
\end{aligned}
$$




$$
\begin{aligned}
& \gamma_{x x x y}^{T}=\gamma_{x x x y}^{M} \sqrt{\frac{\left(\mu_{x x}^{M}\right)^{3}\left(\mu_{y y}^{M}\right)}{\left(\mu_{x x}^{M}-\mu_{x x}^{A}\right)^{3}\left(\mu_{y y}^{M}-\mu_{y y}^{A}\right)}}, \\
& \gamma_{x y y y}^{T}=\gamma_{x y y y}^{M} \sqrt{\frac{\left(\mu_{x x}^{M}\right)\left(\mu_{y y}^{M}\right)^{3}}{\left(\mu_{x x}^{M}-\mu_{x x}^{A}\right)\left(\mu_{y y}^{M}-\mu_{y y}^{A}\right)^{3}}} .
\end{aligned}
$$

In these formulas, the upper index $\mathrm{M}$ corresponds to the measurement results of the primary biased distributions, $\mathrm{A}-$ to the parameters of the instrument function, $\mathrm{T}-$ to the restored turbulence parameters.

\section{Testing of programs by the method of numerical simulation}

The final dimensions of the luminous end of the fiber-optic sensor lead to smoothing and blurring of the true picture of the probability distribution of its displacements. The reconstruction of the true picture in the general case requires the solution of the Fredholm integral equation, which in a simplified symbolic form can be represented as

$$
w M(x, y)=w T(x, y) * w A(x, y)
$$

where $w M(x, y)$ is the measured distribution; the asterisk on the left side of the equality denotes the integral convolution of the required distribution $w T(x, y)$ and the instrument function $w A(x, y)$. FFT algorithms are used to restore the true distribution. An example of numerical modeling of the problem of excluding the instrument function is illustrated in Fig. 2.
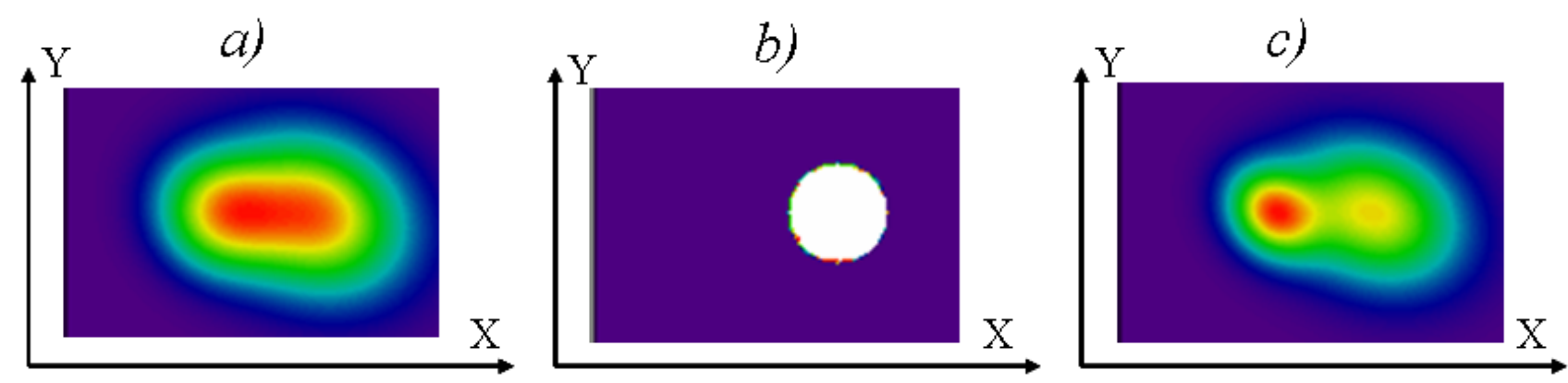

Fig. 2. Rainbow cartograms of simulated FOA signals of the projection type: a) - the signal $w M(x, y)$ before the exclusion of the hardware function; $\mathrm{b})$ - the instrument function $w A(x, y)$ (the signal from the stationary sensor) c) - the reconstructed signal $w T(x, y)$.

The higher moments of the distribution $w T(x, y)$ of interest can be determined directly from the reconstructed signal and compared with the results of calculations using the formulas of the previous section. This allowed us to test the developed algorithms and programs. The use of relations between moments is more preferable, since the reconstruction procedure is unstable if there are even insignificant noises in the measured distributions.

\section{Measurements in a turbulent jet}

The hydrodynamic part of the experimental device is schematically shown in Fig. 3. It allowed creating a jet axisymmetric flow in a water-filled cell with transparent walls. The initial velocity at the outlet of the nozzle was $U_{0}=5 \mathrm{~m} / \mathrm{s}$ and was controlled by a pressure 
indicator (Pitot tube). The nozzle diameter was $d=3 \mathrm{~mm}$, which corresponded to the turbulent flow regime with the Reynolds number for the nozzle diameter $R e=15000$. A sensor with a diameter of 120 microns and a length of $7 \mathrm{~mm}$ was used. The Reynolds number for the sensor diameter at the flow rate $U_{0}=5 \mathrm{~m} / \mathrm{s}$ was $\operatorname{Re}=600$.

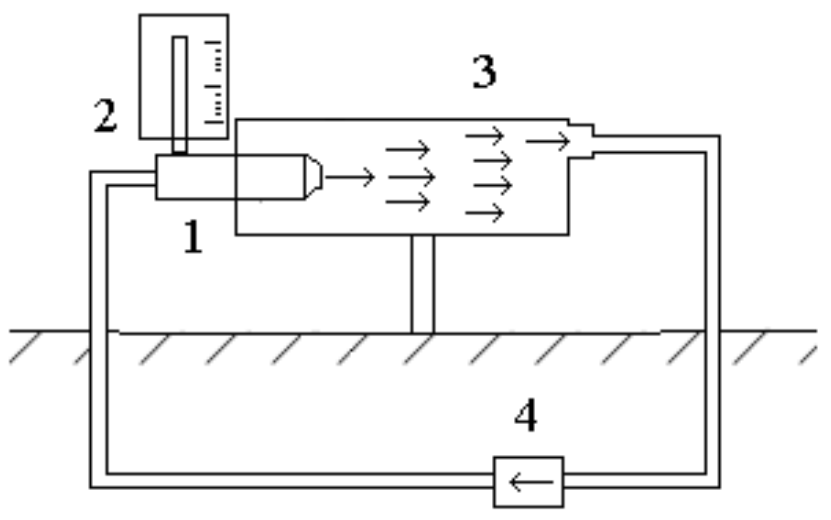

Fig. 3. Hydrodynamic circuit: 1 - a pipe with a profiled nozzle for forming a fluid flow jet; 2 - a Pitot tube; 3 - a cuvette filled with water, 4 - the pump.

An example of the results of FOA measurements in the transverse axial section of a turbulent jet is shown in Fig. 4 .

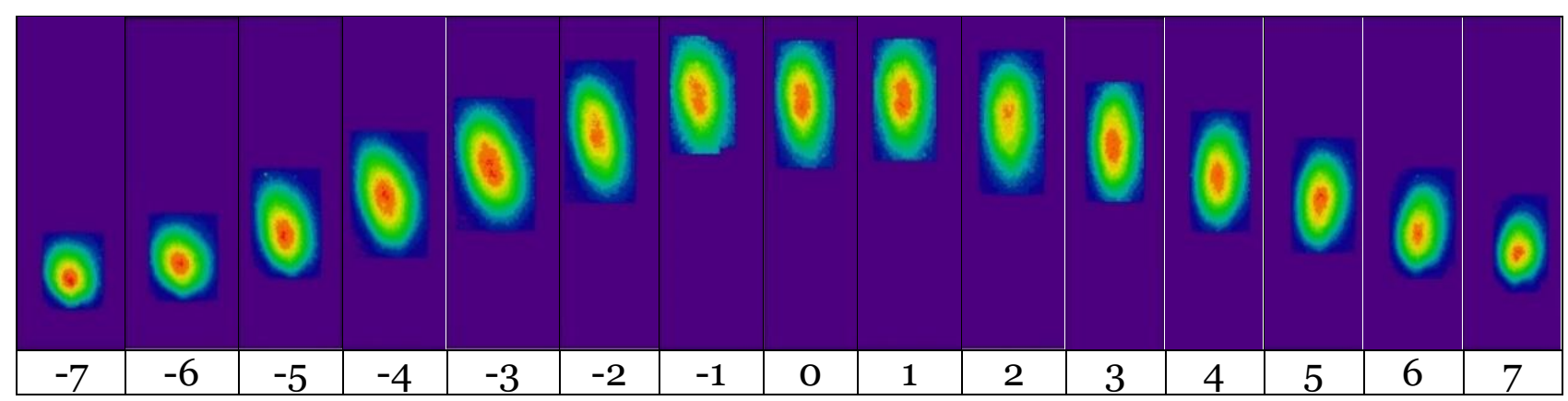

Fig. 4. An example of the FOA of measurements at the points of the cross-section of a turbulent jet (a rainbow color scale is used).

The images shown in Fig. 4 can be interpreted as a convolution of the two-dimensional probability density of the flow velocity at a given point with the instrument function of the fiber sensor. According to the formulas presented in the above, it is possible to calculate the two-dimensional moments of turbulent pulsations.

\section{Parametric models}

The convolution of a circular function and a two-dimensional Gaussian function was used as a three-dimensional model of the average velocity field. The parameters were selected in such a way that in the initial region of the flow, the profile in the axial section was close to rectangular, and then smoothly transformed into Gaussian.

$$
\begin{gathered}
U(r, z)=\frac{1}{T} \int_{0}^{T} u(r, z, t) d t \\
U(r, z)=A(z) \operatorname{circ}\left[\frac{r}{r_{0}}\right] * \exp \left[-\frac{r^{2}}{[B(z)]^{2}}\right], \\
r=\sqrt{x^{2}+y^{2}}
\end{gathered}
$$


The $z$ axis is directed along the flow axis, $r_{\mathrm{o}}$ is the initial radius of the jet.

As a three-dimensional model of the mean square pulsation axial velocity

$$
\sigma_{u}(r, z)=\sqrt{\frac{1}{T} \int_{0}^{T}[u(r, z, t)-U(r, z)]^{2} d t}
$$

the Gaussian model was used:

$$
\sigma_{u}(r, z)=\frac{1}{C(z)} \exp \left[-\frac{\left(r-r_{0}\right)^{2}}{[D(z)]^{2}}\right] .
$$

To model the field of turbulent shear stresses $\sigma_{u_{v}}(r, z)$, the derivative of the mean flow field along the radius was used.

The type and parameters of the approximating functions $A(z), B(z), C(z), D(z)$ were selected from the conditions of simplicity and minimum deviations from the experimental data used [10].

\section{Visualization of fields of turbulent parameters}

For a visual quantitative representation of the fields of turbulence parameters, we then use the "Fire" color scale, shown in Fig. 5.

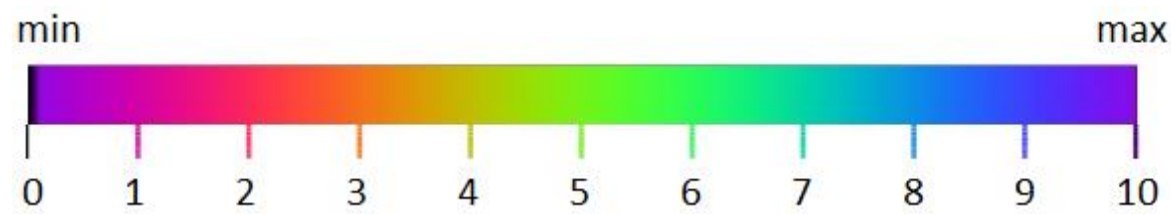

Fig. 5. The "Fire" color scale.

The results of visualization of the average velocity field in the axial section of the jet are shown in Fig.6. Figure 7 gives a visual representation of the transformation of the twodimensional profile of the average velocity in the cross section along the flow axis.

The field patterns and transverse profiles of the RMS pulsation velocity are shown in Fig. 8 and Fig. 9 .

Fig. 10 shows the field of shear turbulent stresses.

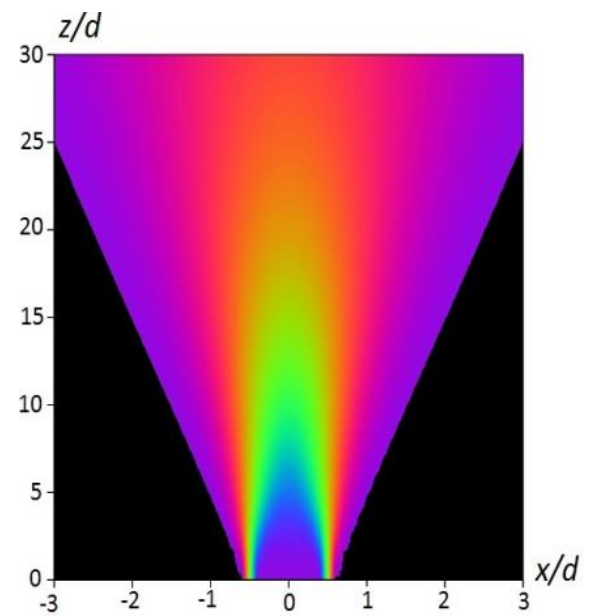

a)

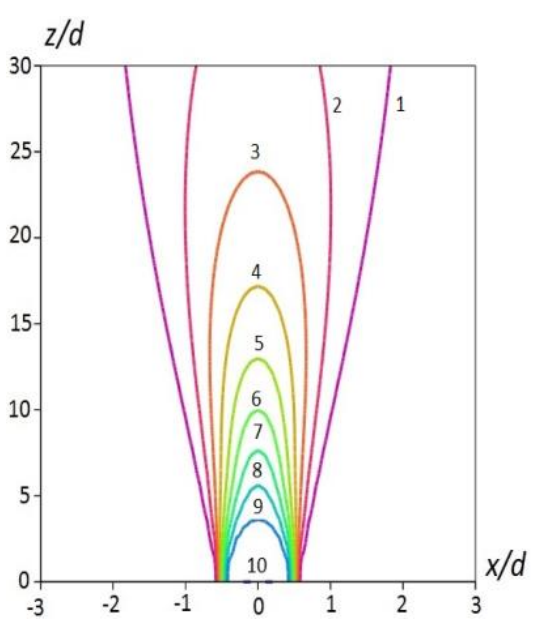

b)

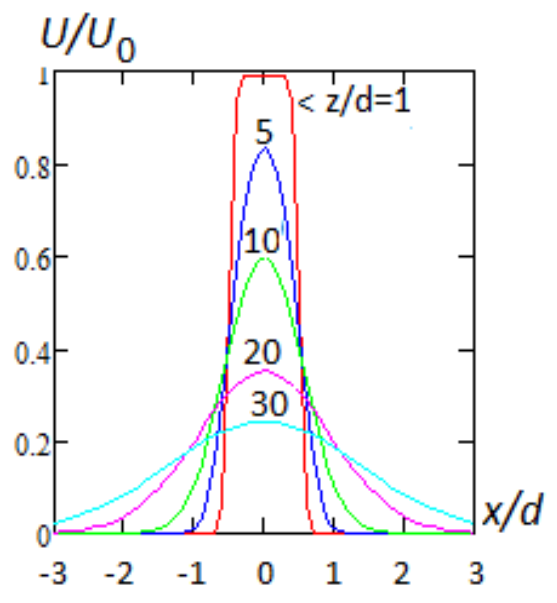

c)

Fig. 6. a) Map of the average velocity field; b) Lines of the same average velocity;

c) Average velocity profiles in transverse axial sections. 


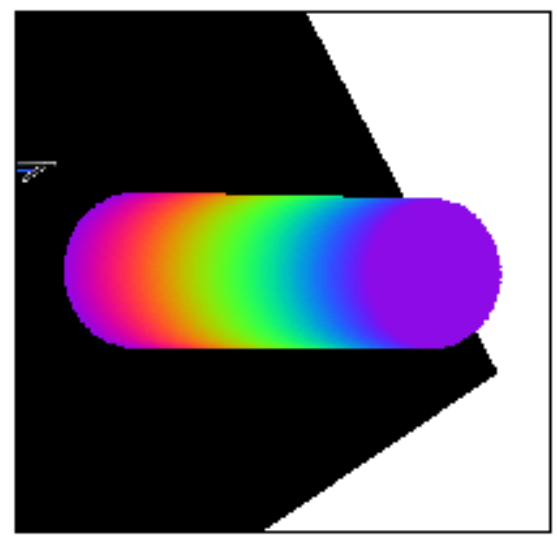

a)

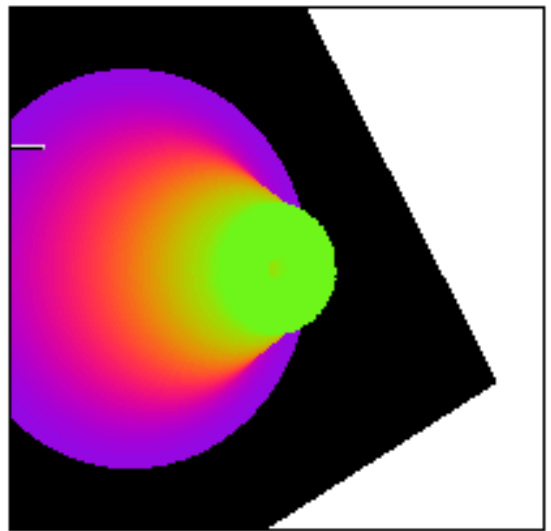

b)

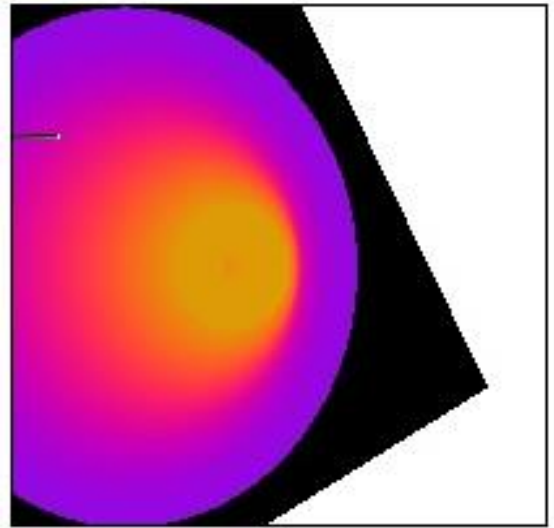

c)

Fig. 7. 2D profiles of the average velocity in various cross-sections of the jet: a) $z / d=0$; b) $z / d=15$; c) $z / d=30$.

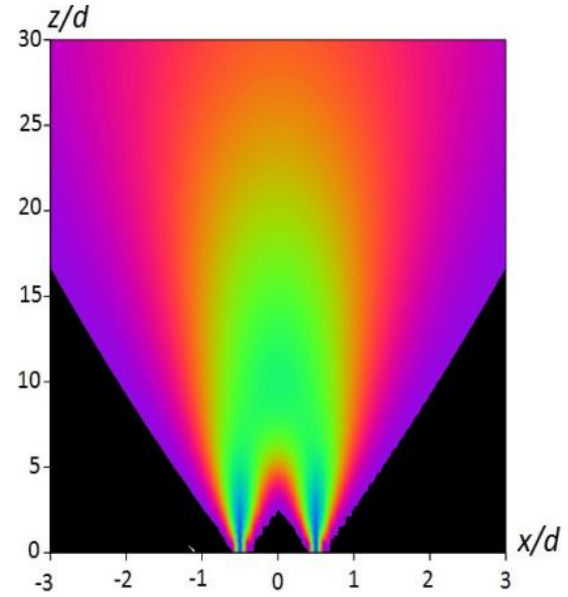

a)

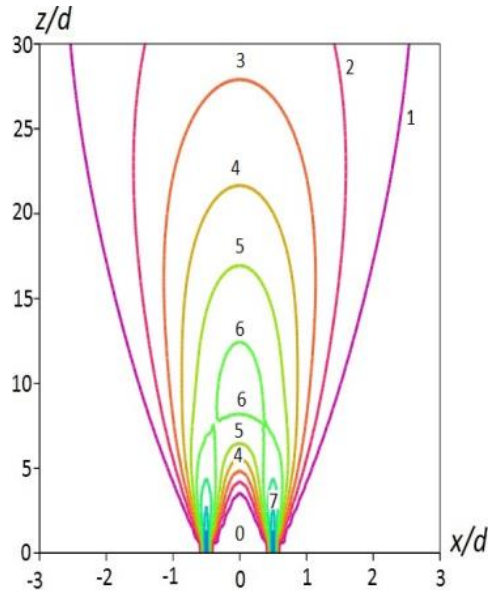

b)

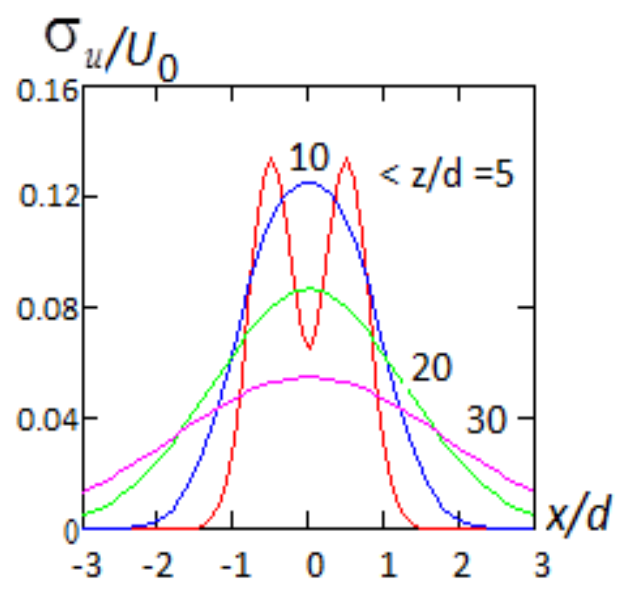

c)

Fig. 8. a) A map of the field of root-mean-square pulsations; b) Lines of equal rootmean-square pulsations; c) Profiles of root-mean-square pulsations in transverse axial sections.

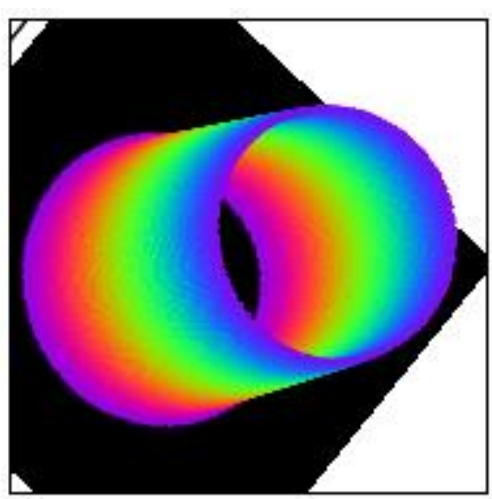

a)

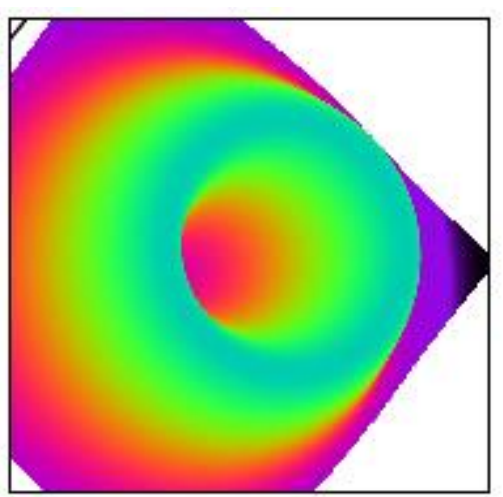

b)

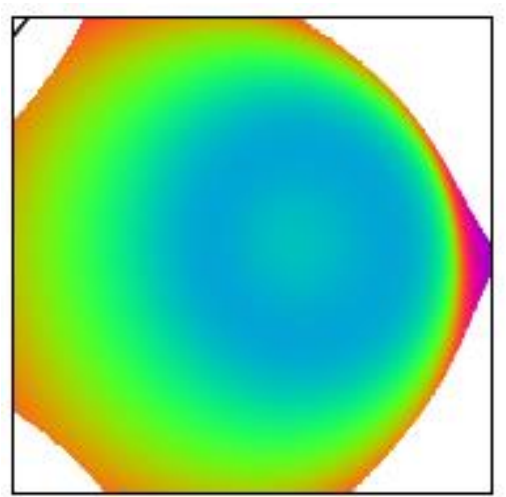

c)

Fig. 9. 2D profiles of root-mean-square velocity pulsations in various cross-sections of the jet: a) $z / d=2$; b) $z / d=8$; c) $z / d=15$. 


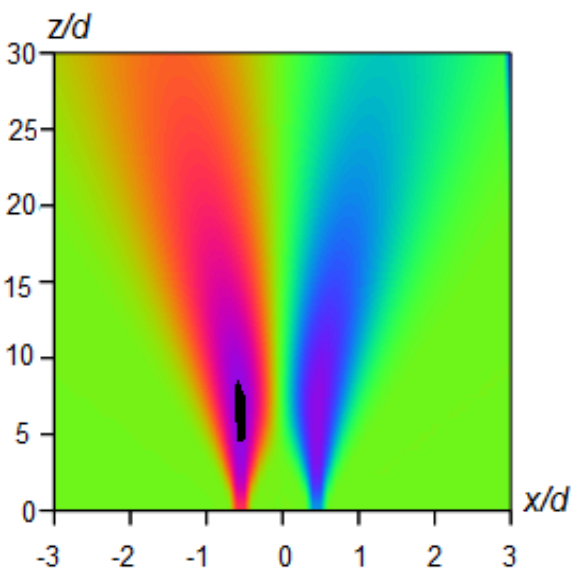

a)

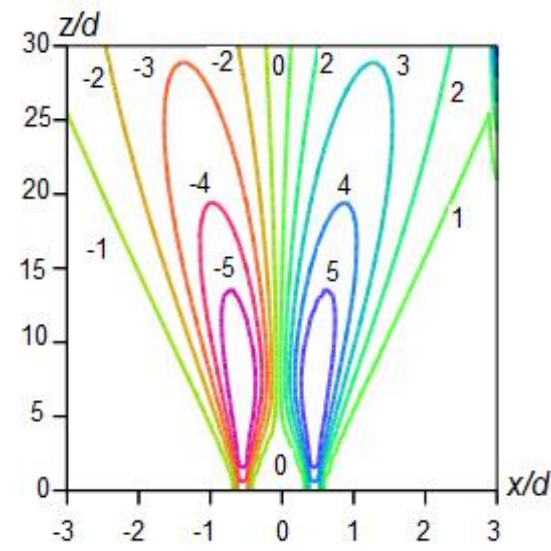

b)

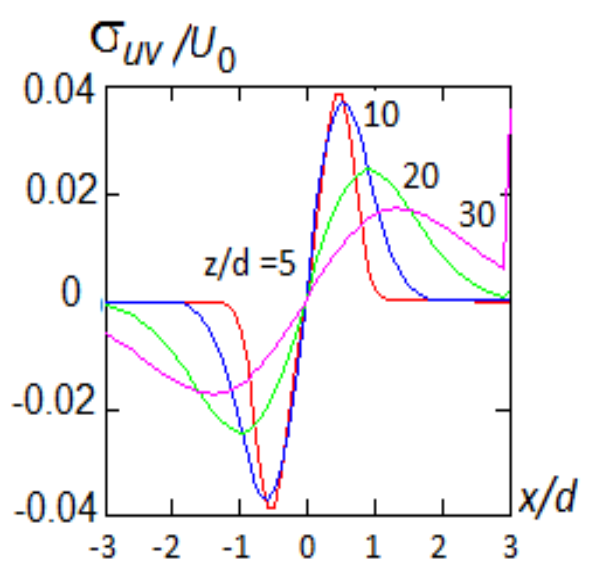

c)

Fig.10. a) A map of the shear stress field; b) Lines of equal shear stresses; c) Profiles of shear stresses in transverse axial sections.

It should be noted that the presented results for the root-mean-square pulsations of velocity and shear stresses in the initial region of the jet $(z / d<3)$ are very conditional, since the developed turbulence has not yet been formed here.

The animations below show pictures of turbulence parameter fields in various color maps (Fire, Rainbow, Neon, Royal, Topography).

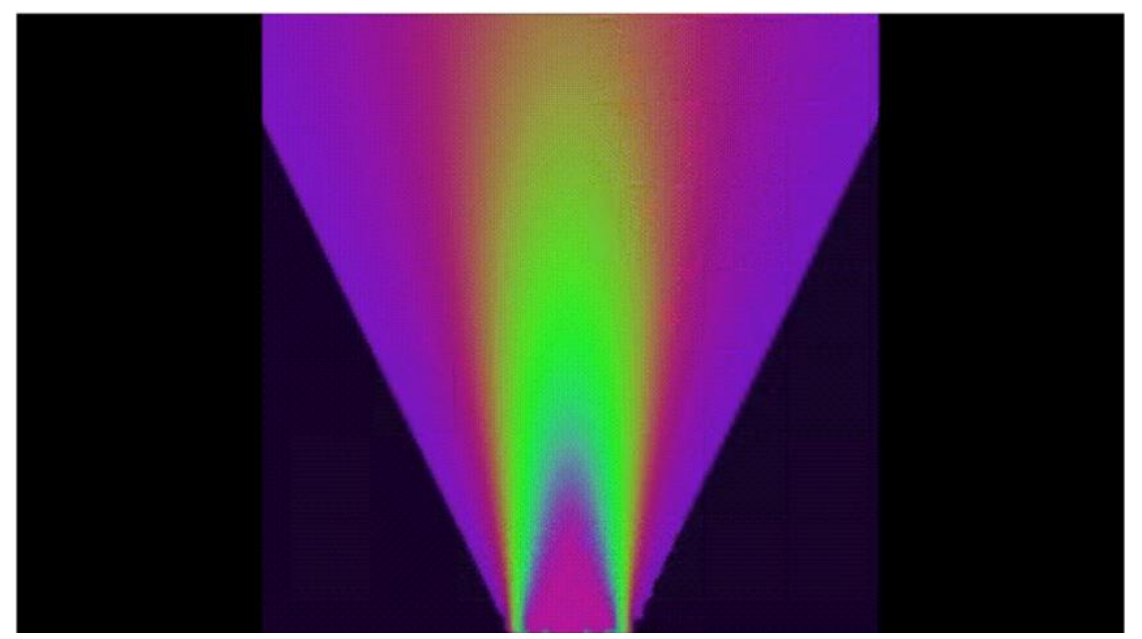

Animation 1. The average velocity field in different color maps.

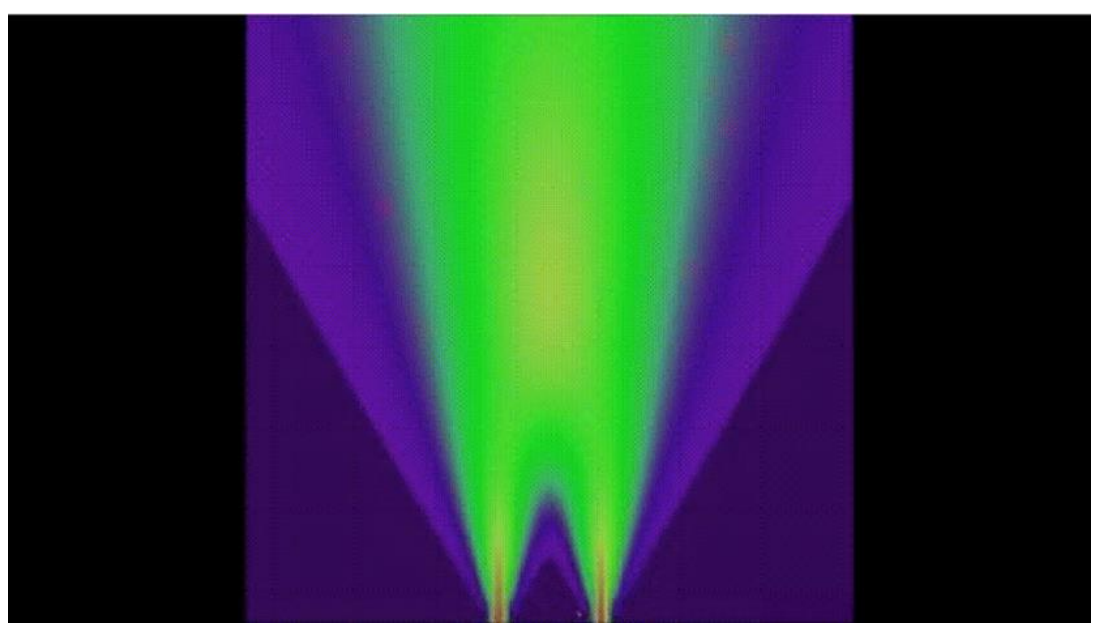

Animation 2. The field of root-mean-square pulsations in different color maps. 


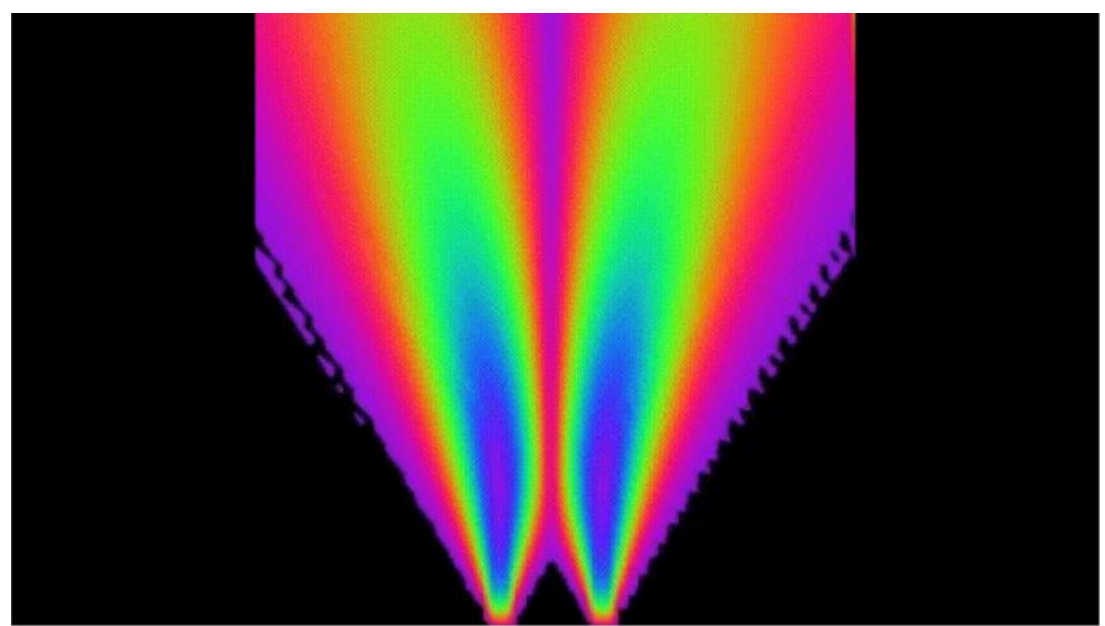

Animation 3. The modulus of the shear stress field in different color maps.

An example of the measurement results of the shape coefficients is shown in Fig.11.

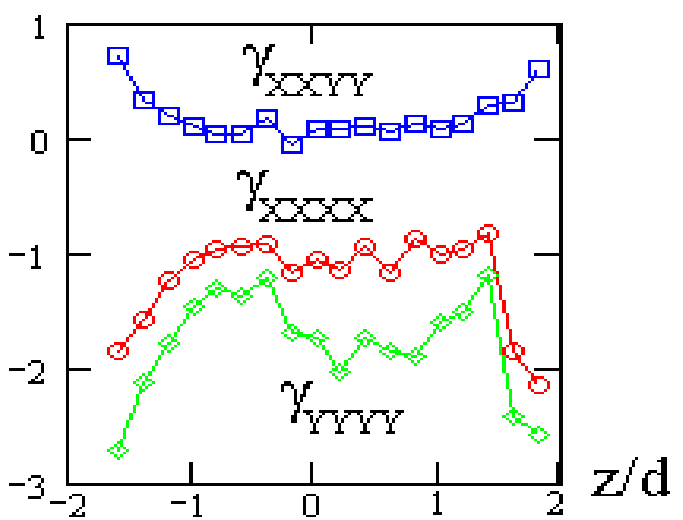

Fig. 11. Measurement results in the axial cross-section $x / d=5$ : profiles of the kurtosis coefficients $\gamma_{\mathrm{XXXX}}, \gamma_{\mathrm{YYYY}}, \gamma_{\mathrm{XXYY}}$.

Deviations of the pulsation statistics from the normal law for the near-axial regions may be due to the presence of coherent vortex structures in the flow. Intermittency and return flows caused by the peculiarities of the cuvette geometry and its finite dimensions can affect the peripheral regions.

The influence of methodological errors caused by the optical-mechanical design of the sensor cannot be excluded.

\section{Conclusion}

A method of computer visualization of the fields of turbulence parameters based on local measurements at individual points of the flow is developed. Simple parametric models are proposed to describe the fields of turbulence parameters in an axisymmetric jet stream. The results of quantitative visualization are presented, which make it possible to calculate the energy dissipation and other integral characteristics of the flow.

\section{References}

1. Abramovich G. N. Theory of turbulent jets. M.: Fizmatiz. 1978.

2. Belotserkovsky S. M., Ginevsky A. S., Khlapov N. V. Modeling of a circular turbulent jet by the method of discrete vortices, Dokl. RAS, 345:4(1995),479-482. 
3. Smirnov V. I. Laser diagnostics of turbulence: dissertation ... doctors of Physical and Mathematical Sciences: 01.02.05. - Moscow, 1997. - 258 p.: ill. RSE OD, 71 99-1/7-8

4. Rinkevicius B. S. Laser Doppler anemometry. - M.: Knorus. 2017.

5. Loginov A. A. Smirnov V. I. Three-beam two-channel computerized laser Doppler measurements of turbulence characteristics in a liquid jet. // Measurement Techniques. 1996. No. 8. pp. 35-40.

6. Biryukova O. V., Smirnov V. I. Measurement of statistical characteristics of turbulence by an optical system with a hybrid fiber-optic sensor. // Measurement Techniques. 2006. No. 9. pp. 25-29.

7. Kondakov I. A., Smirnov V. I. Multi-sensor fiber-optic turbulence sensor. Proceedings of the IX International Scientific and Technical Conference. conf. Optical methods of flow research. Moscow: MEI. 2007. pp. 510-513.

8. Kondakov I. A., Panyukov R. A., Smirnov V. I. Investigation of the operation of a laser fiber-optic turbulence sensor in a liquid jet. Proceedings of the XI International Scientific and Technical Conference. conf. "Optical methods of flow research" Moscow, June 27-30, 2011. Electronic version of the proceedings of the conference. $6 \mathrm{p}$.

9. Kerv Yu. V., I. A. Kondakov I. A., Smirnov V.I. Laser fiber-optic measurements of two-dimensional statistical characteristics of turbulence of the 3 rd and 4 th order $\backslash \backslash$ Optical methods of flow research: XIII International Scientific and Technical Conference [Electronic resource]: proceedings of the conference. - Electron. dan. - Moscow: NRU "MEI", 2015. pp. 512-517 - - ISBN 978-5-00086-752-5

10. Bard Y. Nonlinear estimation of parameters. M.: Statistics. 1979. 